\title{
Geometric Perturbations and Asymmetric Vortex Shedding about Slender Pointed Bodies*
}

\author{
Scott M. Murman \\ ELORET \\ Moffett Field, CA 94035 \\ smurman@nas.nasa.gov
}

\begin{abstract}
The flow about slender, pointed bodies can be characterized by different states with angle of attack. At moderate-to-high angles of attack $\left(\alpha \approx 40^{\circ}\right)$, a steady, asymmetric vortex pattern develops along the body, leading to a net lateral force. At higher angles of attack $\left(\alpha \approx 60^{\circ}\right)$, the aft-end of the body develops an unsteady von Kármán shedding. As the angle of attack approaches $90^{\circ}$, the entire body length exhibits a time-dependent vortex shedding pattern. The current work uses three-dimensional, thin-layer Navier-Stokes simulations to investigate the physical mechanisms of asymmetric vortex shedding at $\alpha=40^{\circ}$ and $\alpha=60^{\circ}$. The development of an asymmetric vortex pattern via a convective instability mechanism is investigated using tip bumps, surface roughness, and tip curvature. It's found that surface roughness simulations can incite an asymmetric vortex state at $\alpha=60^{\circ}$ which is consistent with the application of a tip bump. and the experimentally observed flowfield. The unsteady von Kármán vortex shedding on the aft portion of the body is also well resolved. The use of surface roughness did not incite a flow asymmetry at $\alpha=40^{\circ}$, and it was necessary to simulate tip curvature at this angle of attack in order to generate an asymmetric vortex state.
\end{abstract}

\section{Introduction}

It is well known that the wake behind a cylindrical body aligned perpendicular to the oncoming flow can develop an alternating pattern of strong vortices. This alternate shedding of vortices from the body results in an instantaneously asymmetric flowfield, while in the mean* the flowfield remains symmetric. The alternate shedding

* Copyright (C)2000 by the American Institute of Aeroncutics and Astronautics, Inc. No copyright is asserted in the United States under Title 17, C.S. Cocle. The U. S. Government has a royalty-free license to exercise all rights under the copyright claimed herein for Governmental purposes. All other rights are reserved by the copyright owner.

*mean in this work refers to time-averaging. 
of vortices from a circular cylinder is commonly ceferred to as von Kairmain vortex shedding, or a von Kámán vortex street. If a slender, pointed body of circular crosssection, such as a cone-cylinder or ogive cylinder, is plared at an angle of attack relative to the oncoming flow. it's possible to observe a steady asymmetric flow state relative to the lateral symmetry plane of the body. This introduces the counterintuitive notion that a nominally axisymmetric body can develop an asymmetric flow, not just instantaneously as in the flow about a circular cylinder in crossflow, but in a time-averaged sense*

The flow about a pointed, slender borly of revolution at angle of attack can thus be broken down into several (experimentally-observed) [2-9] regimes (cf. Fig. 1.). The angles-of-attack where these flow regimes transition is dependent upon a number of factors, including body geometry, Mach number, Reynolds number, etc. At relatively low angles of attack, roughly $\alpha \leq 20^{\circ}$, the flowfield remains symmetric with respect to the lateral symmetry plane of the body, even if the flow is perturbed slightly. The flow in this regime may remain attached, or boundary laver crossflow separation can occur if the angle of attack is sufficient, leading to a pair of vortices on the leeward side of the body which are symmetric relative to the lateral symmetry plane. At the opposite angle of attack extreme, $70^{\circ}<\alpha \leq 90^{\circ}$ (again approximately), the flow about the length of the body exhibits von Kármán vortex shedding. In between these extremes, it's possible for a nominally symmetric body of revolution to develop a nonzero mean lateral force. In the low-moderate angle-of-attack range, $20^{\circ}<\alpha \leq 40^{\circ}$, the variation of mean lateral force with roll angle forms a continuous periodic curve. In this regime, as the angle of attack is increased, the maximum absolute value of the lateral force also increases. As the angle of attack is increased into the moderatehigh range, $40^{\circ}<\alpha \leq 70^{\circ}$, the variation of lateral force with roll angle can become nearly a square-wave, and the case of a symmetric mean flow becomes very difficult to achieve. This can be referred to as a "bi-stable" regime, or a bi-stable state; the lateral force becomes independent of roll angle except for a "switching" from positive to negative (and vice versa) as a critical roll angle is passed.

Many of the previous experimental investigations noted that small changes near the tip of the model had a large effect on the observed vortex shedding pattern. Degani and Schiff [10], Degani [11], and Degani and Tobak [7-9] proposed that imperfections near the tip of the model could incite a convective instability in the flow, leading to the development of steady asymmetric vortex patterns at high angles of attack. A convective instability is a sub-class of more general instability responses (cf. [12]). This is shown schematically in Fig. 2 as the response to an impulsive perturbation. The behavior in Fig. $2 \mathrm{a}$ is stable, in that the response decays in all directions with time. Fig. $2 b$ shows an example of an absolutely unstable flow. Here the response to the initial disturbance grows in both the upstream $(x / t<0)$ and downstream $(x / t>0)$ directions. A convectively unstable behavior shows growth

*It is also possible for a nominally symmetric body to exhibit a steady asymmetric flow state in crossflow due to an asymmetric boundary layer transition to turbulence(cf. Ref. [1]), however the current work is only concerned with asymmetric vortex states. 

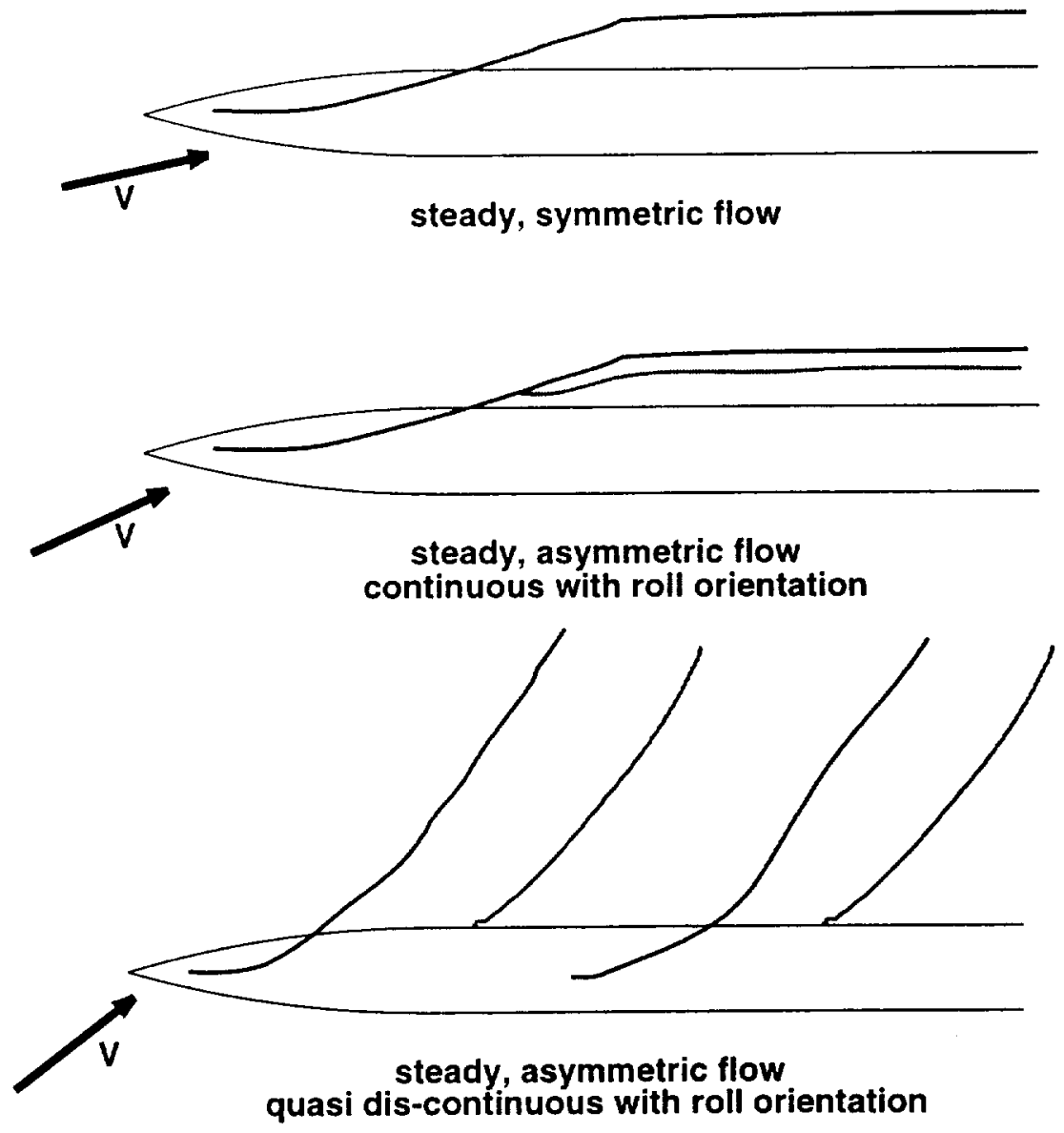

quasi dis-continuous with roll orientation

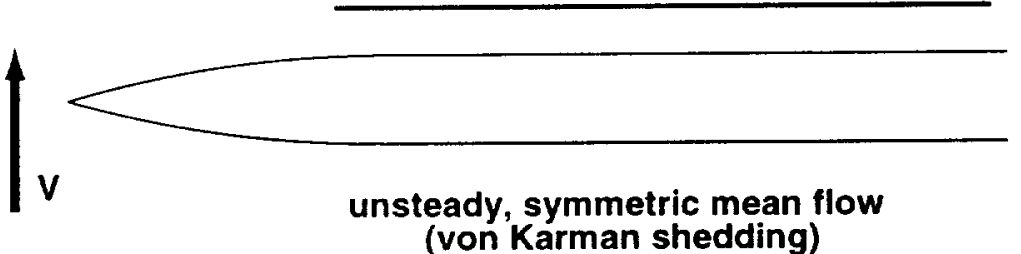

Figure 1: Schematic of flow states about slender pointed bodies. The shaded lines represent vortices rotating in opposite directions.

only in a downstream traveling packet. Given this response, if the perturbation is removed, the flow eventually reverts back to the state before the perturbation was applied.

Computational Fluid Dynamics (CFD) simulations have been used to demonstrate the possibility of a convective instability mechanism for these class of flows. These 


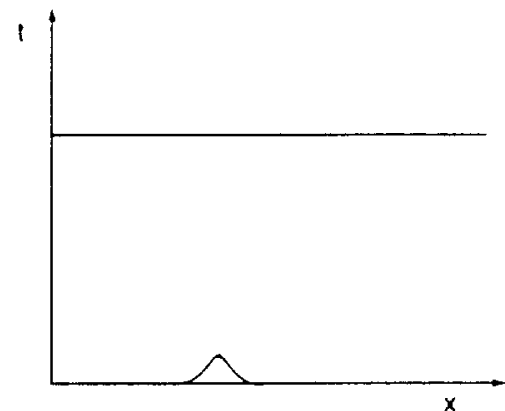

(a) Stable

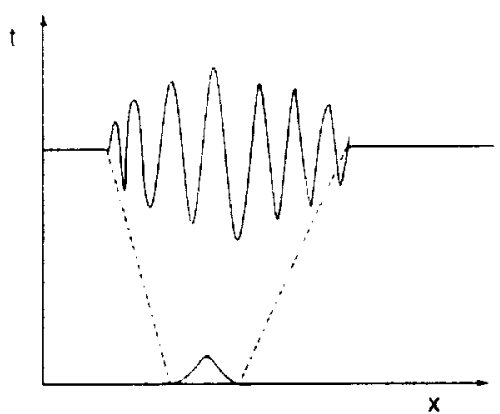

(b) Absolutely Unstable

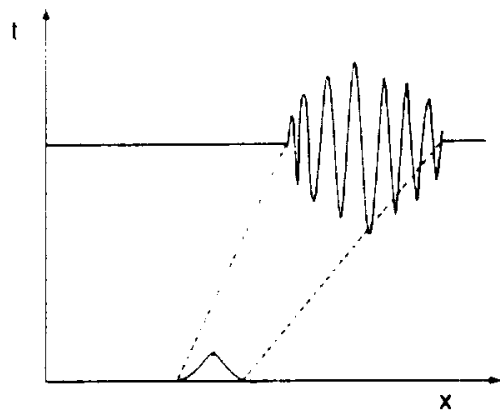

(c) Convectively Unstable

Figure 2: Schematic of responses to an impulsive perturbation.

investigations have primarily emphasized using the controlled environment of a simulation to perform numerical experiments that cannot be performed in a wind tunnel. By perturbing the geometry near the tip of the body, Degani et al. [11.13] and Levy et al. [14] were able to demonstrate that the flowfield in the low-moderate angle-ofattack range behaves as if it possesses a convective instability. In the work of Degani et al., an asymmetric bump perturbation was applied to the tip of an ogive cylinder body until an asymmetric flow state developed, then removed, and the flow reverted to the symmetric state that existed before the perturbation was applied. Levy et al. noted that in the low-moderate angle of attack range increasing the size of the perturbation led to an increase in the flow asymmetry, consistent with the idea of a convective instability. Similar investigations in the moderate-high angle-of-attack regime [7] have indicated that the onset of von Kármán vortex shedding in this regime may be due to an absolute flow instability, although the numerical results are more difficult to interpret in this angle-of-attack regime. Some researchers have been able to simulate an asymmetric mean flow in the moderate-high regime without perturbing the flowfield in any manner $[15,16]$, however these simulations used conical flow, or other simplifying assumptions, which do not allow for the possibility of a convective instability.

In Ref. 17, the author found that ogive cylinders placed at $40^{\circ}$ angle of attack would exhibit an unsteady variation in normal force at $R e_{D}=80,000$ and 200,000, where $D$ is the diameter of the cylindrical portion of the body. These variations were occurring at harmonics of the Strouhal frequency predicted by the Independence Principle, i.e. $S t_{\alpha}=S t_{2 D} \sin \alpha$, and hence were incorrectly related to a possible von Kármán shedding mechanism. Subsequent investigations [18] of crossflow separation in two and three-dimensions, and at several Reynolds numbers using varying grid densities, showed that these unsteady variations were caused by an instability in the free shear layer shed from the body. In a physical flow, these free shear layer instabilities would eventually transition and lead to turbulence in the wake behind the body, as examined experimentally by Roshko [19] and later by Bloor [20]. In 
Rot. 1T, it was alse noted that the anuplification of romnd-off errors was sufficient to lead to a von Kárman vortex shedding along the length an ogive cylinder at $\alpha=60^{\circ}$

and $R_{D}=2(00,000$, and no geometric or other flow perturbations was required to initiate this state.

The current work uses CFD simulations to investigate the physics of vortex shedding about pointed slender bodies; both the steady, asymmetric vortex patterns at moderate angles of attack, and the unsteady, von Kármán vortex shedding that occurs at high angles of attack. Since a large database of experimental results exists for steady asymmetric vortex shedding for Reynolds number $R e_{D}=10,000-200,000$, the current work is performed in this same Reynolds number range. While it has been established that imperfections near the tip of a pointed body can incite a convective instability in the flowfield leading to an asymmetric vortex pattern, the physical mechanisms by which this convective instability affects the changes on the flowfield are still not well understood. Without a detailed understanding of the physical mechanisms involved, designers seeking to eliminate or harness the lateral control power of asymmetric vortex shedding are left to test and develop concepts based solely on physical intuition. The current work seeks to provide insight into the physical mechanisms involved by performing controlled numerical experiments comparing different methods of perturbing the flow; tip bumps, surface roughness, and tip curvature.

\section{Approach}

\subsection{Numerical Algorithm}

This work deals with numerical modeling of two- and three-dimensional separated flowfields. In order to accurately simulate the physics of these flows, it is necessary to use the Navier-Stokes equations as a physical model. The complete Navier-Stokes equations are often simplified using the "thin-layer" approximation [21] when performing high Reynolds number flow simulations. Neglecting the crossflow contributions to the viscous stress tensor still provides satisfactory results for the computed mean drag, and von Kármán shedding frequency at the flow conditions of interest in this work (cf. Ref. 18), and all of the simulations in the current work utilize this simplification to reduce the computational costs. At the Reynolds number considered in the current work, the boundary layer remains laminar, and a numerical turbulence model is not required.

In order to accurately resolve the viscous stress terms, a fine grid spacing is required in each coordinate direction that the viscous stresses cannot be neglected. This grid spacing makes the use of implicit methods desirable when using finitedifference algorithms because of the stiffness of the Navier-Stokes equations. The implicit schemes used in this work are all based on performing a time-linearization of the non-linear flux vectors, and obtaining a so-called "delta formulation" of the governing equations [22]. The backward Euler implicit time-integration scheme is 
used throughout. This is a firsteroder ancurater scheme in time. howewer the allowable numerical time-step due to stability considerations for these fows is much smaller than required to resolve the physics of the unstealy von Karman shedding. and at first-order scheme has been found to provide satisfactory results (cf. Refs. 17. 18).

In order to numerically integrate the governing equations. the spatial derivatives must be replaced by suitable finite-difference formulas. A straight-forward approach is to replace the inviscid derivatives with second-order, central-difference operators, and the viscous derivatives with centered, second-order mid-point operators. Unfortunately, this produces a scheme which is moderately unstable in three dimensions [23]. This weak instability can be overcome however with the addition of numerical dissipation of an order that doesn't interfere with the accuracy of any physical mechanisms. The explicit dissipation is scaled by the local flow properties to reduce it's influence within the boundary layer, where the natural viscous dissipation of the Navier-Stokes equations is suitable.

This combination of central-differenced spatial terms and higher-order numerical dissipation leads to what is referred to here as a block form of the Beam and Warming algorithm $[22,23]$. The block formulation is preferred over the diagonalized algorithm of Pulliam and Chausee [24] due to an inherent asymmetry of the diagonalization process that can lead to spurious flow asymmetries for high-angle-of-attack simulations (cf. Ref. 25).

\subsection{Computational Grids}

Computations were performed about a tangent-ogive cylinder configuration with a 3.5 diameter forebody attached to a 7.0 diameter cylindrical aft section. This is the same configuration examined computationally by Degani et al. [7-9,11], in previous work by the author [17], and experimentally by Lamont $[5,6]$. In previous simulations of vortex shedding about a circular cylinder in crossflow and a tangent-ogive cylinder at $\alpha=60^{\circ}$ and $\alpha=80^{\circ}$, it was found that the von Kármán vortex shedding frequency was not well predicted at $R e_{D}=80,000$ or $R e_{D}=200,000$ (cf. Ref. 17). Grid refinement studies [18] determined a minimum crossflow resolution $\left(\Delta \phi=1.0^{\circ}\right.$, where $\phi$ is the circumferential spacing) required in the separated flow region in order to predict the von Kármán shedding frequency, mean drag, and crossflow separation location at these flow conditions. This recommended crossflow resolution is utilized in this work. This leads to a grid with a crossflow plane having 249 circumferential and 58 radial points for a full-body (periodic) configuration, and 69 points in the axial direction. A typical axial grid plane is shown in Fig. 3 (every third grid line is shown for clarity). The outer boundary of the computational domain is located fifteen body diameters from the surface. At the outer boundary a characteristic boundary condition is applied, while at the downstream exit plane a zeroth-order extrapolation of the flow quantities is performed, which is consistent with the highly convective nature of the flowfield. 


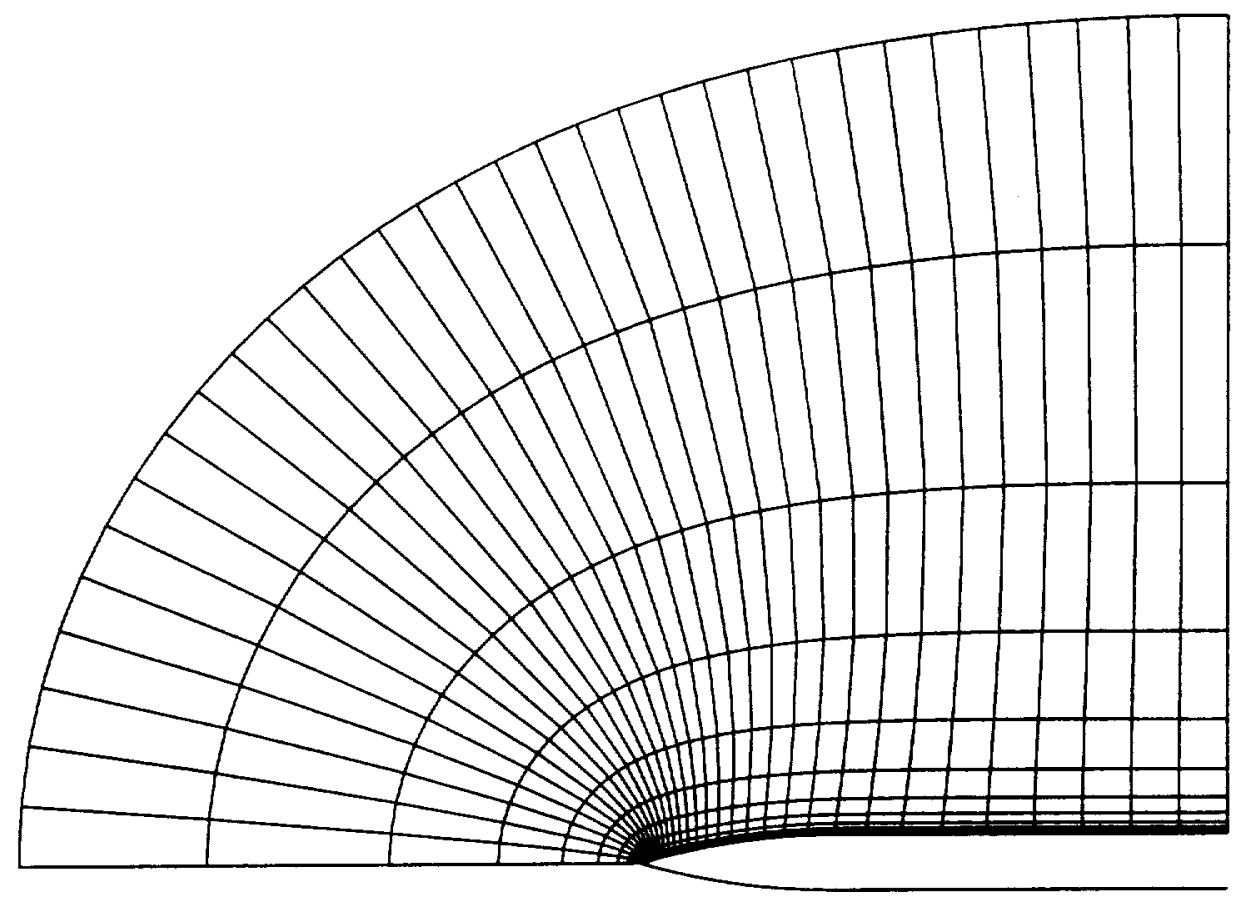

Figure 3: Lateral symmetry plane of the tangent-ogive cylinder computational grid.

\section{Numerical Results}

\subsection{Tip Bump}

As was described in Sec. 1, the flow about a pointed slender body at moderate-tohigh angles of attack can develop a steady asymmetric vortex pattern which produces a non-zero mean lateral force on the body. Previous CFD studies $[11,13,14]$, were able to simulate an asymmetric vortex pattern on a slender body by placing a bump on the body very close to the tip of the nose. In [17], a similar numerical simulation was performed at $\alpha=60^{\circ}$ and $R e_{D}=200,000$. This simulation utilized a similar crossflow resolution $\left(\Delta \phi=3.0^{\circ}\right)$ as the previous studies by Degani et al. and Levy et al. The tip-bump simulation from [17] will be used as a baseline for the current work, and hence is first briefly described.

The computed time-histories of the lateral and normal forces are plotted in Fig. 4. Initially, the geometry was unperturbed, and a von Kármán vortex shedding pattern develops along the length of the borly. This can clearly be seen in the variation of lateral force. The computed vortex shedding frequency and the circumferential location of the crossflow separation are not in good agreement with experimental 
investigations of shedding from a two-dimensional cylinder (cf. Ref. 18). At $t=266$, where $\bar{t}$ is the non-dimensional time, a bump of height $h_{\text {max }}=0.01 d$, where $d$ is the local diameter, was placed at $r / D=0.06$ and $90^{\circ}$ from the windward symmetry plane (cf. Appendix A). After the simulation hiad progressed through several cycles. the perturbation was removed at $\bar{t}=424$.

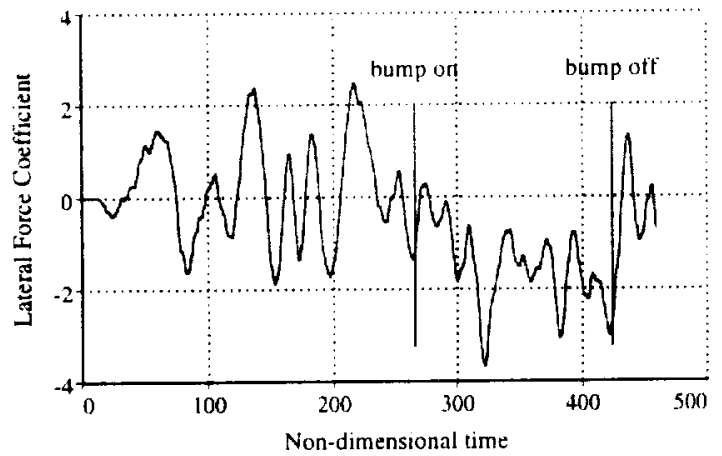

(a) Lateral force

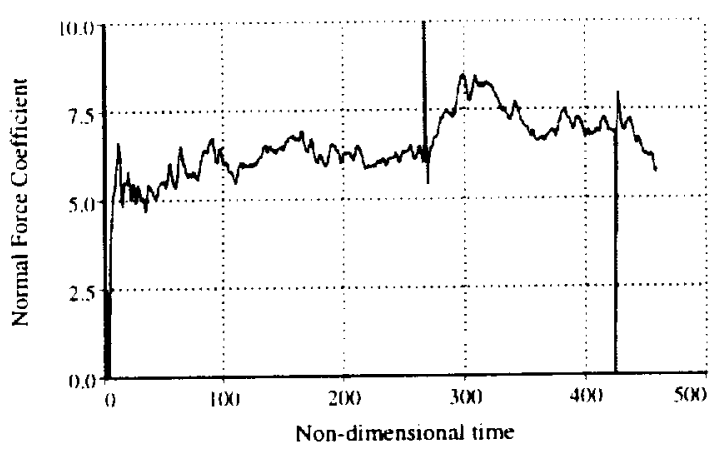

(b) Normal force

Figure 4: Tip bump perturbation force coefficient histories $\left(M_{\infty}=0.20, \alpha=60^{\circ}\right.$, $\left.\operatorname{Re}_{D}=200,000\right)$.

When the bump is placed on the nose the flow develops a non-zero mean lateral force, while still maintaining vortex shedding about the aft-end of the body. The presence of the bump also causes the mean normal force to increase. This is consistent with experimental observations (cf. Ref. 4), which note that the greater the lateral asymmetry, the greater the normal force. The computed flowfield without the bump present contains two main vortices on the leeward side of the body that convect at a small angle to the longitudinal axis of the body and alternate in time. Figure 5 shows an instantaneous snapshot of the unsteady streaklines [26] after the tip perturbation has been applied. In the presence of the bump, the tip vortex on the perturbation side $(R)$ quickly curves away from the body, while the vortex on the opposite side (L) remains attached for a short distance, then curves away from the body. This steady, asymmetric pattern of vortices produces the non-zero mean lateral force. This steady vortex pattern is consistent with those observed experimentally [7,27] and previously simulated numerically by Degani [13] using a similar bump perturbation. Downstream of these two steady, asymmetric vortices the flowfield remains unsteady with the tip bump in place.

When the bump is removed, the flowfield reverts to a symmetric time-averaged flow. This is consistent with the presence of a convective instability mechanism, as has been proposed by Degani and Tobak [7-9]. 


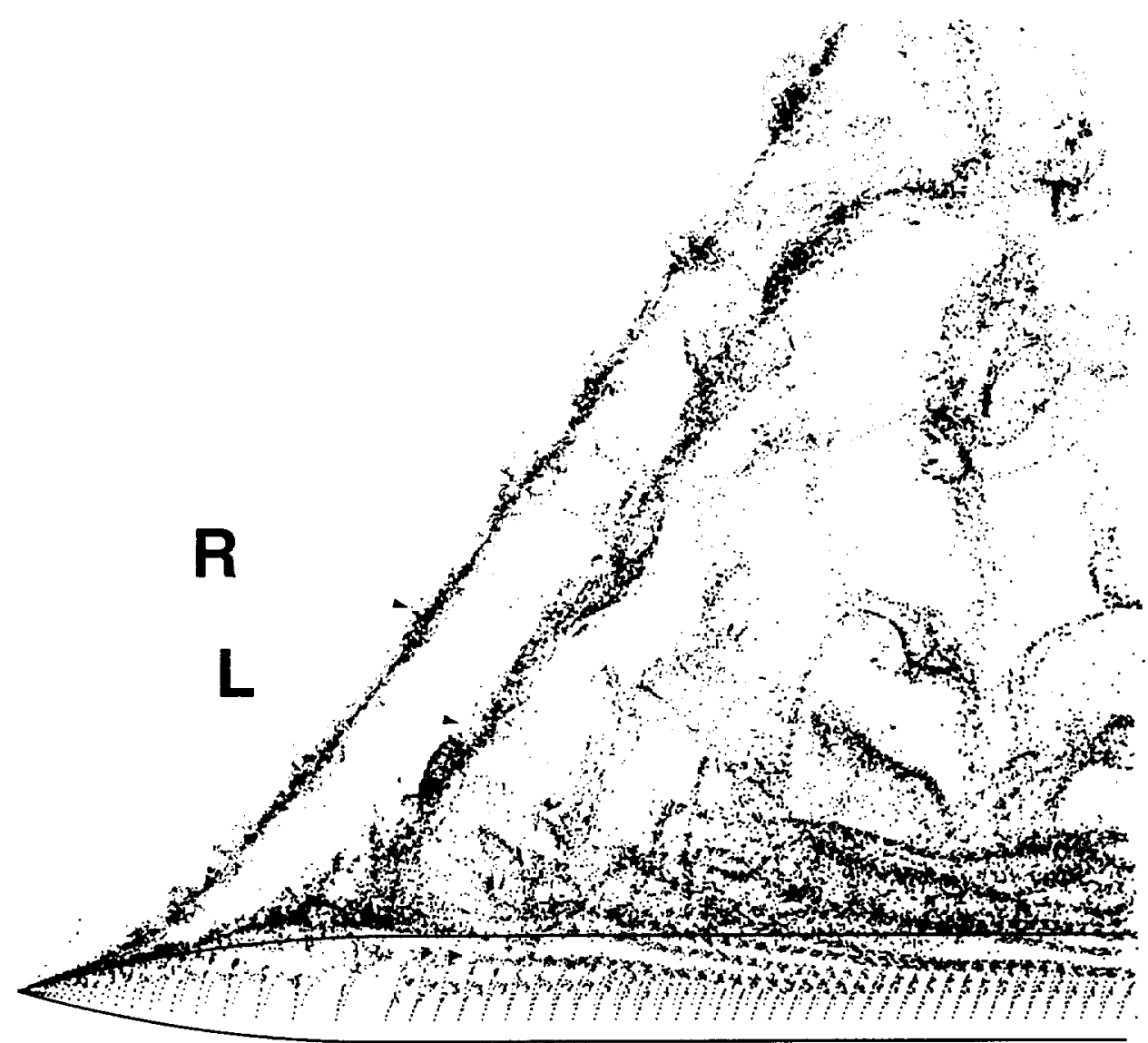

Figure 5: Instantaneous snapshot $(\bar{t}=423)$ of streaklines with tip bump present $\left(M_{\infty}=0.20, \alpha=60^{\circ}, R e_{D}=200,000\right)$.

\subsection{Surface Roughness}

The placement of a fixed bump on the tip of the ogive cylinder is a useful tool for examining the response of the flowfield to a particular perturbation, however it does have limitations. A criticism of the method is that it places a relatively large perturbation (compared to the local radius of curvature) where the flowfield is known to be most sensitive. At low angles of attack it is necessary to use large bump heights to produce the same level of lateral force as observed experimentally (cf. [14]).

The use of a large surface perturbation to "trigger" the convective instability mechanism may effect this change via a different physical mechanism than occurs experimentally. The large perturbation can cause the boundary layer to separate at the bump, and become entrained in the outer, inviscid flowfield. This could bias the flow towards a crossflow separation driven mechanism for asymmetric vortex shedding.

In the current work, it was investigated whether surface roughness can produce the same asymmetric flow states as observed in both previous numerical simulations with fixed tip bumps, and experimentally. The use of surface roughness more accurately mimics the physical geometry encountered with an experimental model, and is easier 
to simulate numerically. The modeling of a bump near the tip can cause problems for both grid generation and numerical statbility. while small surface perturbations are easier to generate and do not appreciably skew the cxisting grid cells.

The flow about a tangent-ogive cylinder at $\alpha=60^{\circ}$ was computed using a crossflow grid resolution of $\Delta \phi=1.0^{\circ}$. The lateral and normal force histories for the simulation are plotted in Fig. 6. The simulation initially had a "clean" geometry, and again the flowfield develops a von Kármán vortex shedding before the perturbation is applied. The variation of lateral force (and normal force) is larger than in the previous tip bump simulation, which is indicative of stronger vortices, and the vortex shedding frequency is lower. Both of these observations are consistent with the increase in crossflow grid resolution in the current surface roughness simulation compared to the prior tip bump studies. The vortex shedding frequency follows very closely the curve $S t_{\alpha}=S t_{2 D} \sin \alpha$ suggested by the Independence Principle. At $\tilde{t}=155.5$ a random distribution of surface roughness with $\Delta_{\max }=1.0 \times 10^{-4}$ was applied (cf. Appendix A). After the surface roughness is applied, the flowfield develops a non-zero mean lateral force in the positive, or pilots-right, direction. The sign of the lateral force (configuration of the asymmetric vortex pattern) would essentially be chosen at random with this method. At $\tilde{t}=228.5$ the surface roughness was removed, and the flowfield reverted back to a mean symmetric flow, again consistent with the behavior of a convective instability.

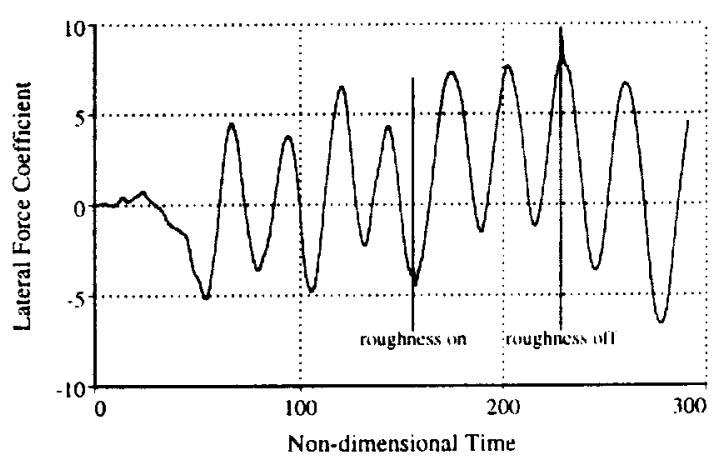

(a) Lateral force

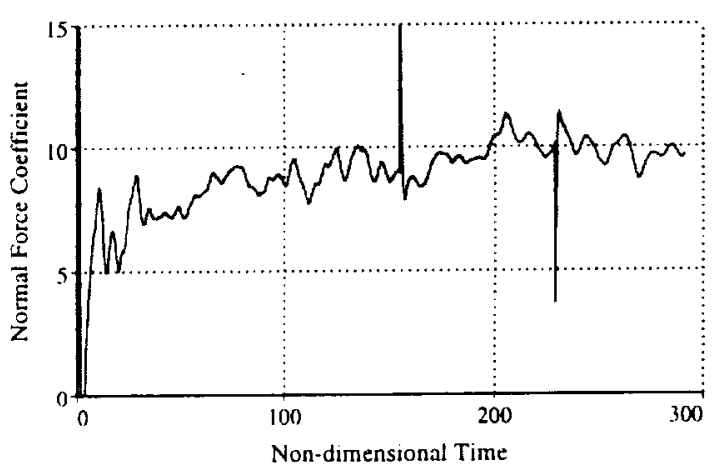

(b) Normal force

Figure 6: Surface roughness force coefficient histories $\left(M_{\infty}=0.20, \alpha=60^{\circ}, \operatorname{Re}_{D}=\right.$ $200,000)$.

Figure 7 shows an instantaneous snapshot of the unsteady streaklines computed with surface roughness applied. As in the bump simulation, one tip vortex (L) lifts rapidly from the body, while the lifting of the tip vortex on the opposite side $(R)$ is delayed. An interface between the space-fixed oblique shedding and the unsteady von Kármán vortex shedding on the aft-end of the body can be seen.

Another useful tool for examining the asymmetric: flowfield, and it's response to perturbations, is the variation of sectional lateral force with both axial distance along 


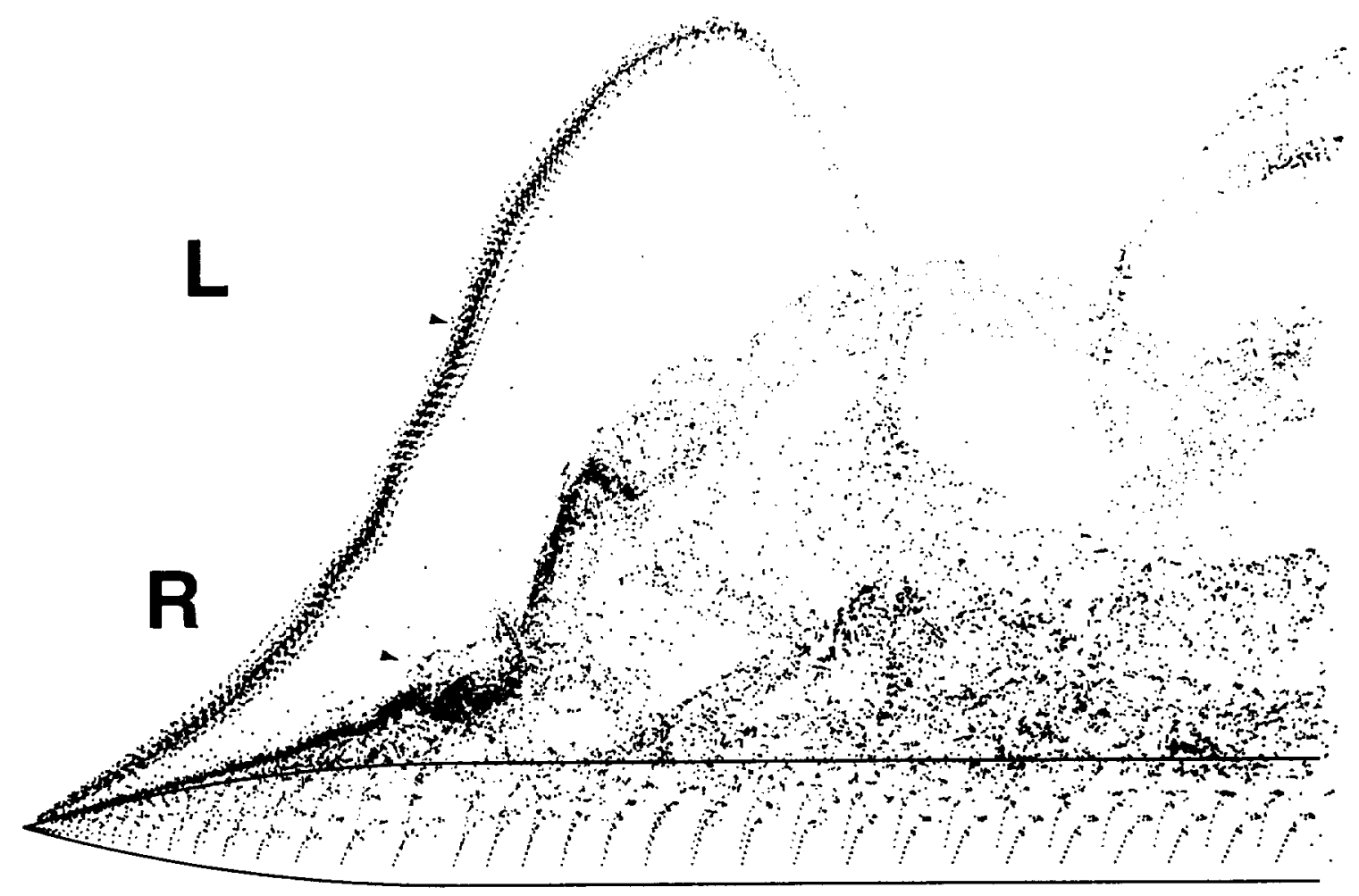

Figure 7: Instantaneous snapshot $(\tilde{t}=205)$ of streaklines with surface roughness present $\left(M_{\infty}=0.20, \alpha=60^{\circ}, R e_{D}=200,000\right)$.

the body and non-dimensional time. This data is presented as a three-dimensional carpet plot with sectional lateral force being the dependent variable. The sectional lateral force variation is plotted for both the tip bump simulation and the surface roughness simulation in Figs. 8 and 9 respectively*. The lateral force maps are similar for the two simulations. In both cases the unsteady vortex shedding is clearly visible on the aft-end of the body $(x / D>6)$. The frequency of the vortex shedding is higher, and the amplitude of the variation lower, in the tip bump simulation, which is consistent with its lower crossflow grid resolution. On the tangent portion of the nose, both simulations show a steady lateral force which originates as a perturbation from the nose tip. In the tip bump simulation the lateral force is negative (towards the pilots left), while in the surface roughness simulation the sign is reversed. Both simulations also show a high amplitude, high frequency variation of lateral force near the nose-cylinder junction $(x / D=3.5)$. In the surface roughness simulation this variation is difficult to see because it's superimposed on the steady lateral force on the nose region. These high frequency peaks may be related to the onset of vortex shedding on the cylindrical portion, and the high frequency shear-laver instabilities discussed in Sec. 1.

*Note that Figs. 8 and 9 do not have the same time scale. Refer to Figs. 4 and 6 to determine the perturbation application for the respective simulations. 


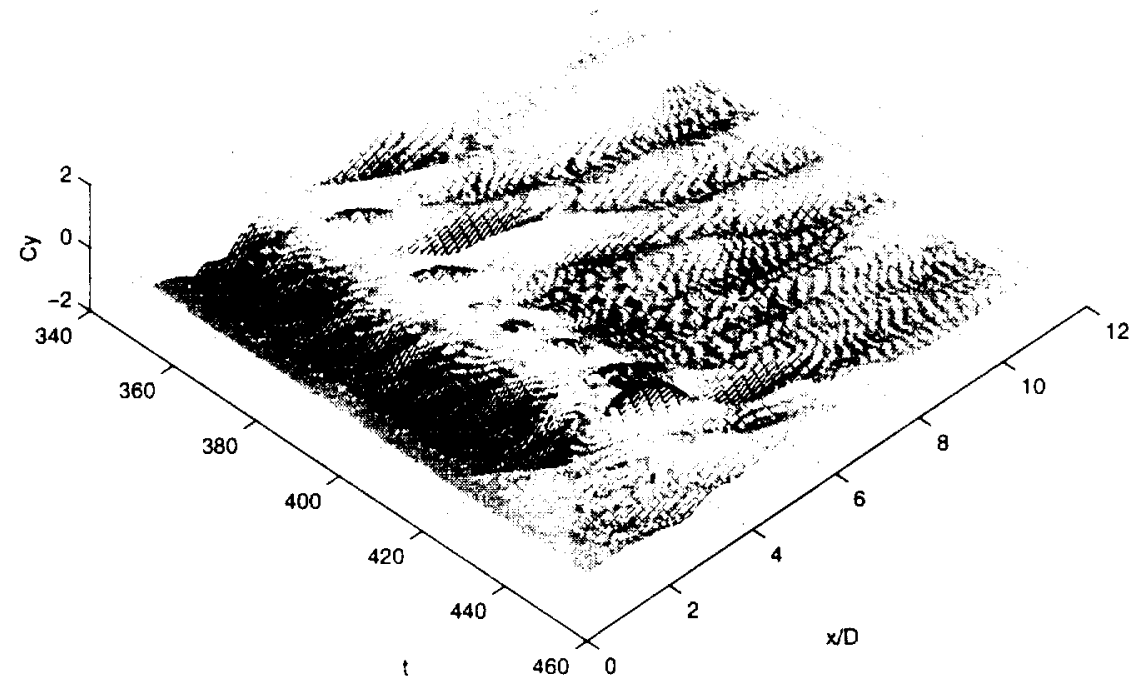

Figure 8: Variation of sectional lateral force with tip bump present $\left(M_{\infty}=0.20\right.$, $\alpha=60^{\circ}, R e_{D}=200,000$ ).

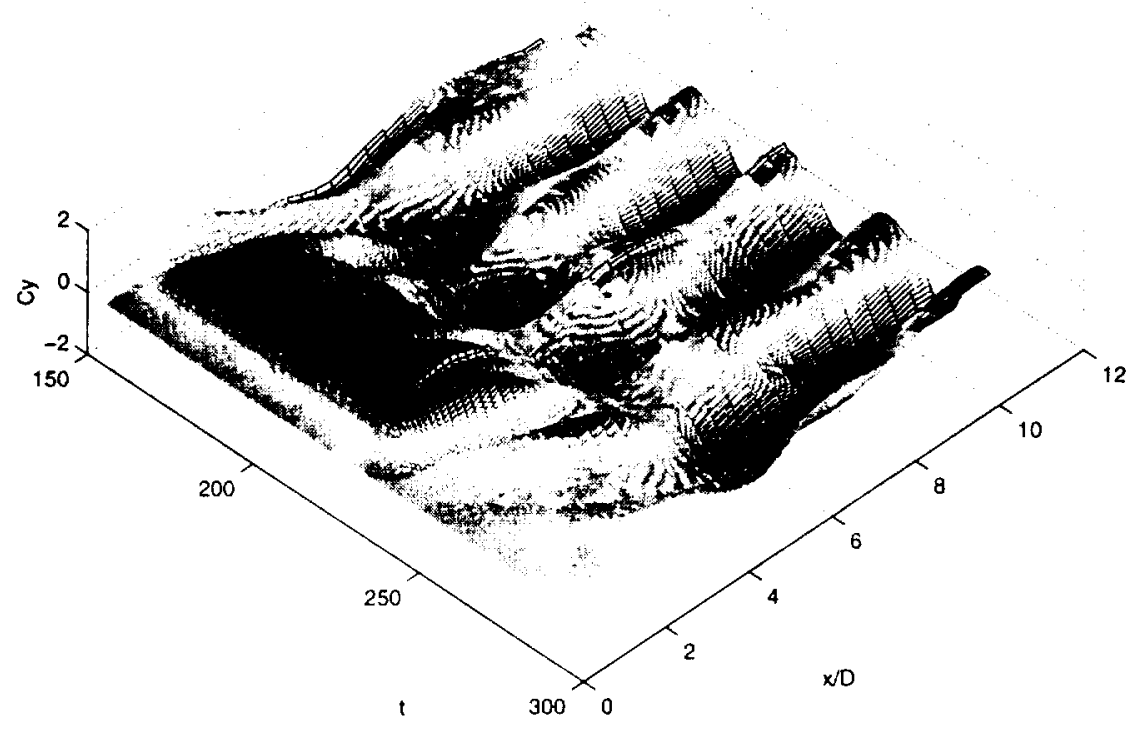

Figure 9: Variation of sectional lateral force with surface roughness present $\left(M_{\infty}=\right.$ $\left.0.20, \alpha=60^{\circ}, R e_{D}=200,000\right)$. 
It isn t possible to see in the resolution of Figs. 8 and 9. but very close to the tip point. the flowfield is highly unsteady in the tip bump simulation, while in the surface roughness simulation the flow is steady (and asymmetric). This unsteadiness is caused by the tip bump forcing a rrossflow separation. Figure 10 contains the helicity density contours in a crossflow plane located at $x / D=0.06$ for both the tip bump and surface roughness simulations. As can be seen, the tip bump causes an irregular, highly unsteady configuration of the main vortices, while the asymmetric vortices in the surface roughness simulation remain steady. The irregularities in the free shear layer due to the shear-layer instabilities in the surface roughness simulation are also visible. The steady asymmetric flow pattern that is observed on the entire forebody in the surface roughness simulation is an indication that the flow has been perturbed (slightly) into a "stable" flow state, not one that is artificially induced.

When the steady asymmetric vortex pattern and the unsteady von Kármán shedding exist simultaneously, it's hypothosized that a front separates the two states, possibly similar to the weak pressure fronts described by Williamson [28]. As seen in the numerical simulations, and through physical arguments (cf. [8]), this front must remain stationary relative to the body (Fig. 11). If the tip perturbation is removed, the new flow state can also be interpreted in the light of a response to a perturbation, in this case removing the tip imperfections. Since the von Kármán shedding appears as an absolute instability, the response moves faster than the body travels, hence the pressure front should move upstream until the entire body experiences unsteady von Kármán shedding.

\subsection{Tip Displacement}

It was attempted to generate an asymmetric flow at $\alpha=40^{\circ}$ without resorting to the use of a tip bump. It was first attempted to utilize the surface roughness simulation that was effective at $\alpha=60^{\circ}$, as described above. Initially, the same roughness height of $\Delta_{\max }=1.0 \times 10^{-4}$ was applied, however this did not generate an appreciable lateral force. The roughness height was increased to $\Delta_{\max }=0.001$ everywhere, yet still it was not possible to generate an asymmetric flow state. Finally, a configuration was created which maintained a roughness height of $\Delta_{\max }=0.01$ on the nose of the body $(x / D<1.0)$ and $\Delta_{m a x}=0.001$ elsewhere, yet this still did not generate an appreciable lateral force on the body at this angle of attack.

Another geometry modification which is consistent with experimental models is an asymmetry of the sharp tip. Moskovitz et al. [29] reported that the tip of their model appeared curved when viewed under a microscope. A configuration was generated where the tip point was offset $0.001 D$ in the $y$-direction from the lateral symmetry plane, while the remainder of the geometry was unmodified. The flow about this geometry was simulated at $\alpha=40^{\circ}$. The lateral force immediately began to diverge from zero after the tip was moved, and eventually the flow reached a steady asymmetric state. A similar effect was found at $\alpha=60^{\circ}$, however a comparable computation to the surface roughness simulation described above has not been completed to date. 


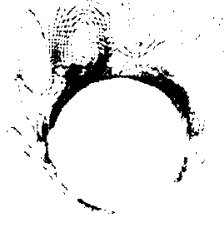

$\tilde{t}=354$

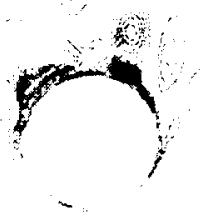

$\tilde{t}=375$

Tip bump

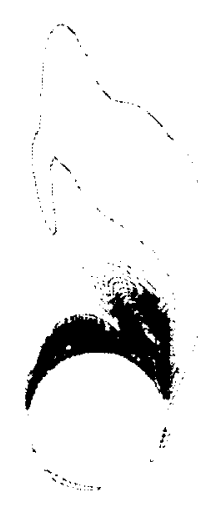

$\tilde{t}=160$

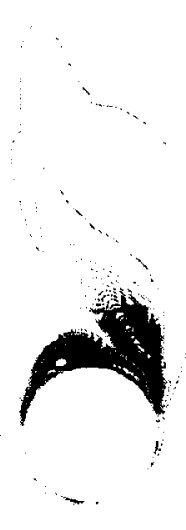

$\tilde{t}=180$

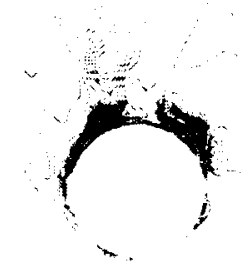

$\tilde{t}=400$

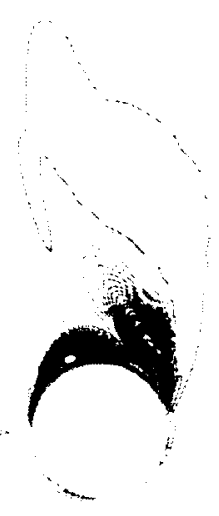

$\tilde{t}=200$

Surface Roughness

Figure 10: Helicity-density contours at $x / D=0.06\left(M_{\infty}=0.20, \alpha=60^{\circ}, R e_{D}=\right.$ $200,000)$.

The effect of the tip displacement on the the lateral force evolution can be traced to the perturbation response being convected down the length of the body. Figure 12 shows the variation of sectional lateral force along the body with time. The response convects downstream creating an asymmetric vortex strength, with the asymmetry in the positive direction for $3<x / D<6$ and in the negative direction for $6<x / D<10$. The final distribution of sectional lateral force is in relatively good agreement with 


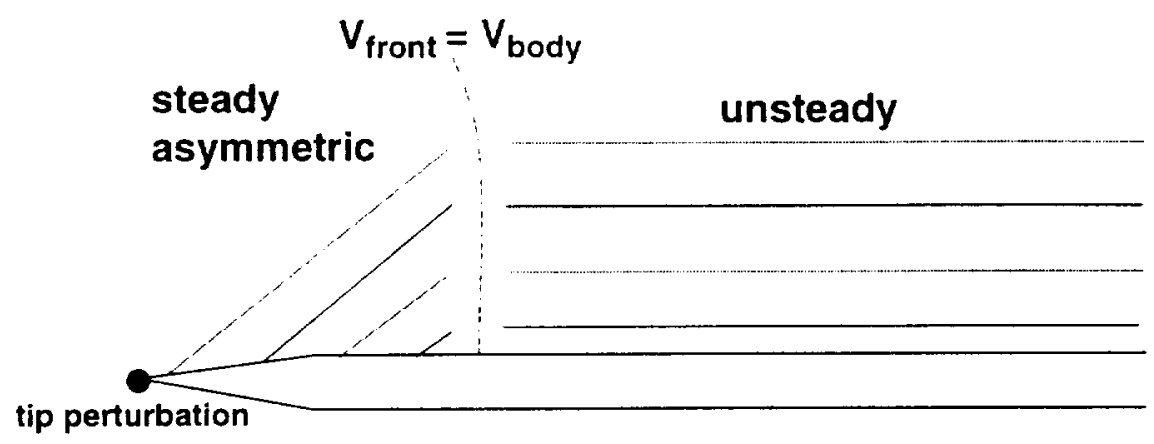

Figure 11: Schematic of front separating steady and unsteady flow regions.

the experimentally observed modes (cf. Refs. 4,6,14).

From the off-surface streamline pattern (cf. Fig. 13) it was noted that the tip displacement in this configuration did not cause an asymmetric oblique vortex shedding pattern to develop, rather the two tip vortices have different strengths on opposite sides of the body. This is a prelude to the asymmetric vortex pattern that can develop, and is consistent with observations of a continuous "sine-wave distribution" of lateral force with roll angle. It was attempted to cause the vortices to "lift-off" the body by increasing the tip displacement. This did lead to a larger flow asymmetry, but the vortices remained nearly parallel to the body longitudinal axis. Similar results were obtained by combining a tip curvature simulation with a surface roughness of $\Delta_{\max }=0.001$ everywhere, and combining tip curvature with a slight sideslip angle $\left(\beta=2.0^{\circ}\right)$. The trends observed (that a larger perturbation leads to a larger asymmetry) are consistent with previous numerical results from Levy et al. [14] using tip bumps to perturb the geometry. Levy et al. were able to cause the vortices to lift off the body at this angle of attack, however in that case the flowfield was fully turbulent, which may affect the behavior. Similar to the results obtained using surface roughness simulations, utilizing a tip displacement did not cause the crossflow separation location near the tip of the body to become unsteady.

\section{Summary}

Simulations of asymmetric vortex states about a tangent-ogive cylinder configuration at high angles of attack were performed using three means of geometrically perturbing the body; tip bumps, surface roughness, and tip curvature. It is seen that the tip bump does provide a useful tool for simulating the cumulative effects of tip asymmetries, however it can artificially excite the asymmetric state, especially near the tip of the nose. A method of simulating surface roughness was found to produce a steady asymmetric state on the forebody of the ogive cylinder at $\alpha=60^{\circ}$, with unsteady von Kármán shedding on the aft end of the body. At $\alpha=40^{\circ}$, the surface roughness was ineffective in producing an asymmetric vortex configuration, however a method of simulating tip curvature did produce a pair of leeward vortices 


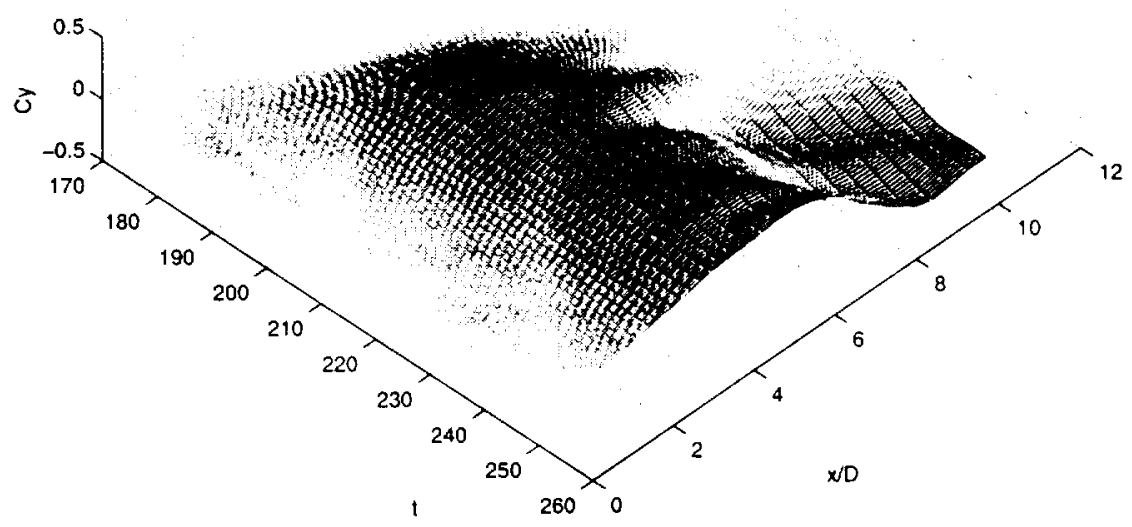

Figure 12: Variation of lateral force with tip displacement present $\left(M_{\infty}=0.20\right.$, $\alpha=40^{\circ}, R e_{D}=200,000$ ).

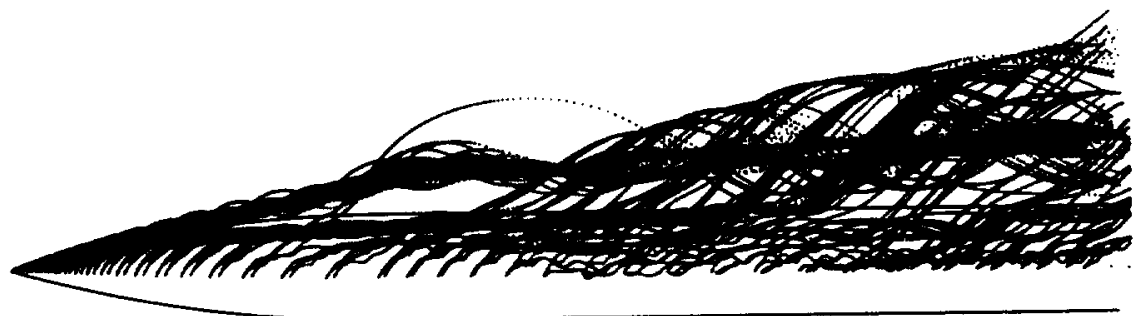

Figure 13: Off-surface streamlines with tip displacement present $\left(M_{\infty}=0.20, \alpha=\right.$ $40^{\circ}, R e_{D}=200,000$ ).

with different strengths. Both of these methods are considered "non-intrusive", in that any changes to flowfield near the tip are induced rather than forced, and the flowfield remains steady. However, it was not possible to simulate large flow asymmetries at $\alpha=40^{\circ}$ with the tip curvature method. The use of the surface roughness or tip curvature methods are preferred over the tip bumps as they are simpler to implement numerically, and can be used for detailed investigations into the physical mechanisms causing the vortex asymmetries without forcing the crossflow separation to be displaced.

\section{Acknowledgments}

The author would like to thank Murray Tobak of NASA Ames Research Center for his helpful discussions during the course of this work, and Stuart Rogers, also of NASA 
Ames, for his insightful comments in reviewing the manuscript.

\section{A Geometry Perturbations}

\section{A.1 Bump Geometry Definition}

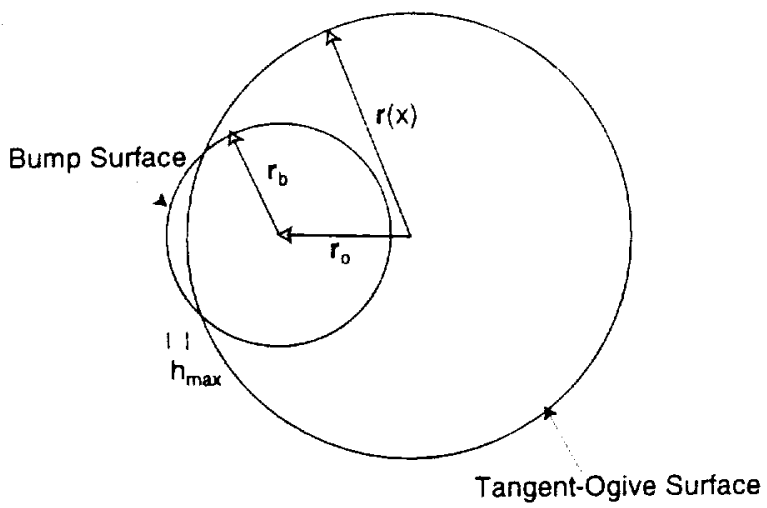

(a) Front view ( $y-z$ plane)

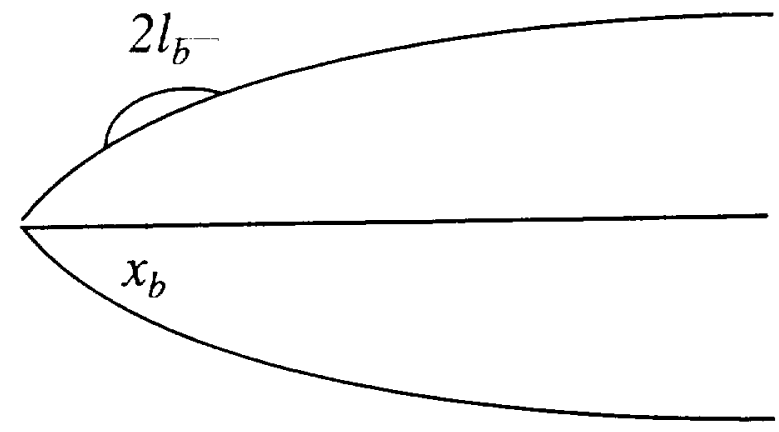

(b) Top view ( $x-y$ plane)

Figure 14: Bump geometry.

The bump geometry is defined as a circular perturbation following Fig. 14. $h_{\max }$ is the maximum bump height, and is placed at an axial location $x_{b}$. The $x$-axis is assumed to coincide with the longitudinal axis of the body. The magnitude of the bump radius, $\left|\boldsymbol{r}_{b}\right|$, is also specified. A value of $\left|\boldsymbol{r}_{b}\right|=0.5|\boldsymbol{r}(x)|$ has been found to give good results for tip bumps. The longitudinal distribution of bump contours is created by defining a bump height distribution in $x$, and building planes of circular bumps, i.e.

$$
h(x)=h_{\max }\left[1-\left(\frac{x-x_{b}}{\ell_{b}}\right)^{2}\right]
$$

Where $\ell_{b}$ is the half-length of the bump perturbation. A value of $\ell_{b}=2\left|\boldsymbol{r}\left(x_{b}\right)\right|$ has given good results with tip bumps. This leaves the origin of the bump circle to be solved from

$$
|\boldsymbol{r}(x)|+h(x)=\left|\boldsymbol{r}_{o}\right|+\left|\boldsymbol{r}_{b}\right|
$$

In the current work the bump was always placed $90^{\circ}$ from the windward symmetry plane.

In this manner the existing grid points $(x)$ in the crossflow plane will be modified only if they are located within the region of the bump that protrudes from the body, 
i.e.

$$
\begin{aligned}
& \text { if }\left(\left|x-r_{o}\right|<\left|r_{b}\right|\right) \text { then } \\
& x=r_{o}+r_{b} \\
& \text { fi }
\end{aligned}
$$

\section{A.2 Surface Roughness Simulation}

The effects of surface roughness were modeled by randomly applying small perturbations to the geometry surface,

$$
\boldsymbol{r}^{\prime}=\boldsymbol{r}(x)(1+\Delta) \quad-\Delta_{\max } \leq \Delta \leq \Delta_{\max }
$$

The maximum possible deviation between successive points is thus $2 \Delta_{\max }$. The perturbation was only applied to half the surface points,

$$
\Delta= \begin{cases}0 & \text { if rand }[0,1]<0.5 \\ \pm \Delta_{\max } \text { rand }[0,1] & \text { otherwise }\end{cases}
$$

where rand $[0,1]$ is a pseudo-random number between 0 and 1 inclusive. The perturbation is also chosen to be positive or negative at random. The random numbers in the above equation (including the \pm test) are considered to be distinct.

\section{References}

[1] Schewe, G., "On the Force Fluctuations Acting on a Circular Cylinder in Crossflow from Subcritical up to Transcritical Reynolds Numbers," Journal of Fluid Mechanics, vol. 133, pp. 265-285, 1983.

[2] Thomson, K. D. and Morrison, D. F., "The Spacing, Position and Strength of Vortices in the Wake of Slender Cylindrical Bodies at Large Incidence," Journal of Fluid Mechanics, vol. 50, no. 4, pp. 751-783, 1971.

[3] Hunt, B.L., "Asymmetric Vortex Forces and Wakes on Slender Bodies," AIAA Paper 82-1336, Aug. 1982.

[4] Dexter, P.C. and Hunt, B.L., "The Effect of Roll Angle on the Flow Over a Slender Body of Revolution at High Angles of Attack," AIAA Paper 81-0358, Jan. 1981.

[5] Lamont, P.J., "The Complex Asymmetric Flow over a 3.5D Ogive Nose and Cylindrical Afterbody at High Angles of Attack," AlAA Paper 82-0053, Jan. 1982 . 
[6] Lamont, P.J., Pressures Around an Inclined Ogive Cylinder with Laminar, Transitional, or Turbulent Separation." AIAA Journal. vol. 20). pp. 1492-1499, Nov. 1982.

[7] Degani, D. and Tobak. M.. "Numerical, Experimental, and Theoretical Study of Convective Instability of Flow ( )ver Pointer Bodies at Incidence," AIAA Paper 91-0291, Jan. 1991.

[8] Degani, D. and Tobak, M., "Effect of Upstream Disturbance on Flow Asymmetry," AIAA Paper 92-0408, Jan. 1992.

[9] Degani, D. and Tobak, M., "Numerical Simulation of Upstream Disturbance on Flows Around a Slender Body," AIAA Paper 93-2956, July 1993.

[10] Degani, D. and Schiff, L. B., "Numerical Simulation of the Effect of Spatial Disturbances on Vortex Asymmetry," AIAA Journal, vol. 29, pp. 344-352, 1991.

[11] Degani, D., "Effect of Geometrical Disturbance on Vortex Assymetry," AIAA Journal, vol. 29, pp. 560-566, Apr. 1991.

[12] Huerre, P. and Monkewitz, P. A., "Local and Global Instabilities in Spatially Developing Flows," Annual Review of Fluid Mechanics, vol. 22, pp. 473-537, 1990.

[13] Degani, D., "Numerical Investigation of the Origin of Vortex Asymmetry," AIAA Paper 90-0593, Jan. 1990.

[14] Levy, Y., Hesselink, L., and Degani, D., "Systematic Study of the Correlation Between Geometrical Disturbances and Flow Asymmetries," AIAA Journal, vol. 34, pp. 772-777, Apr. 1996.

[15] Siclari, M.J. and Marconi, F., "The Computation of Navier-Stokes Solutions Exhibiting Asymmetric Vortices," AIAA Paper 89-1817, June 1989.

[16] Kandil, O.A.,Wong, T.C., and Liu, C.H., "Navier-Stokes Computations of Symmetric and Asymmetric Vortex Shedding Around Slender Bodies," AIAA Paper 89-3397, Aug. 1989.

[17] Murman, S.M., "Computational Study of Shear-Layer Shedding from Ogive Cylinders at High-Angles-of-Attack," AIAA Paper 97-2002, June 1997.

[18] S. Murman, Computational Study of Vortex Shedding about Slender Bodies. PhD thesis, Stanford University, 1999.

[19] Roshko, A.. "On the Development of Turbulent Wakes from Vortex Streets," NACA TR 1191, 1954. 